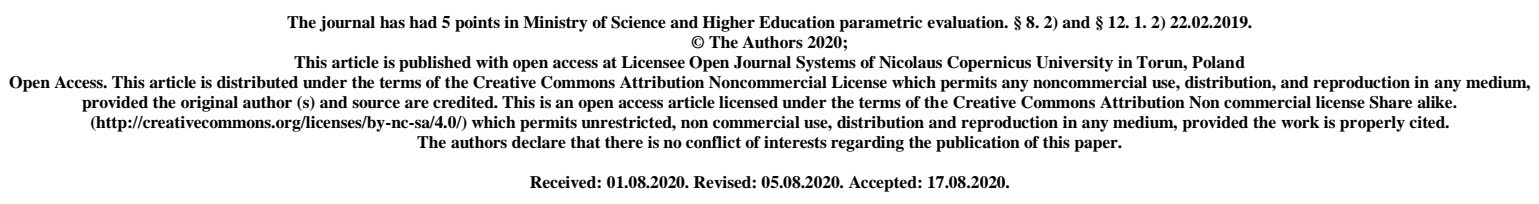

Sirtuin as the target of anti-cancer therapy

\author{
Marzena Baran ${ }^{1}$, Paulina Miziak ${ }^{1}$, Katarzyna Bonio ${ }^{2}$ \\ ${ }^{1}$ Chair and Department of Biochemistry and Molecular Biology, Medical University of Lublin \\ ${ }^{2}$ Department of Cell Biology, Institute of Biological Sciences, Maria Curie-Skłodowska University, Lublin
}

\begin{abstract}
Sirtuins is a group of nicotinamide dinucleotide (NAD +) - dependent, which have deacetylation and ADP-ribosylation activity. They work by detaching the acetyl group from the lysine residue of the histones, thanks to which they influence the control of gene expression. There are 7 classes of sirtuins due to the type of enzymatic activity and the cellular compartment in which they occur. They have a significant impact on the cell cycle, cellular process, including glucose metabolism, fat differentiation, fatty acid oxidation, neurogenesis, carcinogenesis and the aging process. Due to its unique properties, compounds are sought that can control the concentration of sirtuins in the body. One of such substances is resveratrol. It is a naturally occurring polyphenol that has a number of positive effects on the body. It works by increasing the concentration of $\mathrm{NAD}+$, which is a sirtuin cofactor. It occurs naturally, and its greatest concentration is found in grapes and peanuts. It has a beneficial effect in the treatment of pancreatic, colon, breast, prostate and liver cancer. Research indicates that there is a potential for resveratrol supplementation to have positive health effects. However, there is still a need for more research on resveratrol, particularly in animal models. Determining the effectiveness of this chemical as an anti-cancer agent can be a major area of research and clinical trials.
\end{abstract}

Key words: sirtuin, deacetylation, histone deacetylase, resveratrol 


\section{Characteristics of sirtuins}

Sirtuin is a group of proteins belonging to the histone deacetylase family. For their activity, they need a cofactor, which is nicotinamide adenine dinucleotide (NAD) [1]. They have deacetylation and ADP-ribosylation activity [2]. All sirtuins contain a conserved catalytic core domain of 275 amino acids, and differ in length and sequence in the $\mathrm{N}$ - and C-terminal domains $[1,2]$. They are responsible for the regulation of gene expression, control of the cell cycle, cell proliferation and apoptosis, and are responsible for the DNA repair process. Moreover, the activity of sirtuins is connected with longevity. Due to their place of occurrence in cell compartments and activity in humans, 7 groups of sirtuins have been distinguished [1].

Table 1. Characteristics of sirtuins [1,2]

Sirtuins Location in the cell

Enzymatic activityc

Function

Sirtuin 1 Nucleus/ cytoplasm Deacetylase Regulation of metabolism,
immune response, response to oxidative stress, regulation of life expectancy

\begin{tabular}{|c|c|c|c|}
\hline Sirtuin 2 & Nucleus/ cytoplasm & Deacetylase & $\begin{array}{l}\text { Regulation of the cell cycle, } \\
\text { development of the nervous } \\
\text { system }\end{array}$ \\
\hline Sirtuin 3 & Mitochondria & Deacetylase & $\begin{array}{l}\text { Regulation of mitochondrial } \\
\text { metabolism }\end{array}$ \\
\hline Sirtuin 4 & Mitochondria & ADP-ribosyltransferase & $\begin{array}{l}\text { Regulation of mitochondrial } \\
\text { metabolism }\end{array}$ \\
\hline Sirtuin 5 & Mitochondrium & Deacetylase & Apoptosis \\
\hline Sirtuin 6 & Nucleus & $\begin{array}{l}\text { Deacetylase / ADP- } \\
\text { ribosyl transferase }\end{array}$ & DNA repair, genome stabilization \\
\hline Sirtuin 7 & Nucleus & Deacetylase & $\begin{array}{l}\text { Regulation of the cell cycle, } \\
\text { regulation of transcription }\end{array}$ \\
\hline
\end{tabular}

Sirtuins are involved in the programming of cellular metabolism, in particular in glucose metabolism, oxygen glycolysis (Warburg effect), fat metabolism, including fatty acids, cholesterol, acetate and some amino acids metabolism, cell cycle regulation and the formation of neoplastic tumors [3]. The abnormal course of cellular metabolism in neoplastic cells is characterized by increased oxygen glycolysis. It is a key element in the uncontrolled proliferation and growth of a cancerous tumor. At the same time, many types of cancer exhibit increased glutamine metabolism. Moreover, deregulated DNA repair pathways and genome instability favor tumor growth and the acquisition of new mutations [4]. The wide spectrum of sirtuins activities that they participate indicates that these enzymes are involved in the development of metabolic, neurodegenerative, cancer and cardiovascular diseases. Therefore, the regulation of sirtuin activity is a very promising therapeutic target [1]. It has been proven in clinical trials that increased sirtuin 1 levels have a beneficial therapeutic effect. This effect can be achieved thanks to activators such as resveratrol, butein, piceatannol, cambinol. These results lead to the search for synthetic sirtuin activators [5]. 
Sirtuin inhibitors may also be of therapeutic interest as an upregulated SIRT1 regulation has been shown to occur in cancer cells. Reduction of SIRT1 expression may inhibit the proliferation of neoplastic cells [1].

\section{Resveratrol as a sirtuin activator}

Resveratrol (trans-3,4 ', 5-trihydroxystilbene) belongs to the group of polyphenols [6,8]. This compound is naturally present in grapes, wine, peanuts and mulberries [8]. Resveratrol acts as a sirtuin activator by increasing the concentration and co-activator of NAD + [7]. It has been shown that this compound has antioxidant, anti-inflammatory and anti-cancer properties, as well as anti-neurodegenerative and anti-aging properties [6]. Resveratrol shows a favorable correlation with sirtuins [8]. Studies in mice have shown that resveratrol, by increasing the concentration of SIR1, has a beneficial effect on fat metabolism and supports glucose control and increases the sensitivity of cells to insulin [8]. The studies showed that in yeast, an additional copy of the SIR2 gene extends the lifespan, and the aberration of this gene has the opposite effect. Subsequently, it has been found in eukaryotic organisms (nematodes, insects) that manipulation of SIR2 orthologs has a significant effect on life expectancy. Therefore, it can critically affect your life expectancy. It was found that SIR 2 acts as a metabolic sensor that precisely controls gene expression depending on the metabolic state of the cell $[1,8]$. Numerous in vitro studies have shown that resveratrol has multiple anti-tumor effects, protecting against both tumor initiation and cancer progression. Reduces tumor incidence in all species tested to date, including prevention against lymphomas. Resveratrol supplementation in animal cancer models showed positive, neutral, and negative results depending on the route of resveratrol administration, dose, tumor model, species, and other factors. Resveratrol works by inhibiting the cell cycle leading to cell apoptosis, inhibiting the growth and migration of cancer cells. It also acts as an antioxidant which protects the genetic material from damage. Resveratrol inhibits the activity of oxygenase (COX), which plays an important role in tumor formation, and also inhibits the binding of the $\kappa \mathrm{B}$ transcription factor (NF- $\kappa \mathrm{B}$, which can drive the transcription of genes promoting tumor growth $[4,8]$. Resveratrol influences the expression of nurotropic factors of origin. It regulates FOXO1 signaling, which is related to insulin metabolism. Numerous studies indicate that it may inhibit pancreatic cell dysfunction by inhibiting phosphodiesterase activity and inhibit metabolic decline, which is important for diabetics [9] Resweatrol activates sirtuins, thanks to which it reduces the harmful effects associated with aging $[7,9]$. 


\section{References}

1. Villalba J.M, Alcain, Sirtuin activators and inhibitors, Biofactors. 2012 Sep; 38(5), 349-359.

2. Carafa V. i inni, Sirtuin functions and modulation: from chemistry to the clinic, Clin Epigenetics. 2016,8: 61.

3. Zhu S. i inni, The Role of Sirtuins Family in Cell Metabolism during Tumor Development, Seminars in Cancer Biology, 2019, vol. 57.

4. Mai Z. i inni, Sirtuins in metabolism, DNA repair and cancer, J Exp Clin Cancer Res. 2016, 35

5. Dai H. i inni, Sirtuin activators and inhibitors: Promises, achievements, and challenges, Pharmacol Ther., 2018, 88, 140-154.

6. Galiniak S. i inni, Health benefits of resveratrol administration, Acta Biochim Pol, 2019, 66(1),13-21.

7. Lee S-H. i inni, Sirtuin signaling in cellular senescence and aging, BMB Rep., 2019, 52(1): 2434.

8. Kulkarni S. S., Cantó C., The molecular targets of resveratrol, Biochimica et Biophysica Acta (BBA) - Molecular Basis of Disease, 2015, vol. 1852, 1114-1123.

9. McCubrey J.A. i inni, Effects of resveratrol, curcumin, berberine and other nutraceuticals on aging, cancer development, cancer stem cells and microRNAs, Aging (Albany NY), 2017, 9(6), $1477-1536$. 